\title{
ALMOST CONTINUOUS REAL FUNCTIONS
}

\author{
K. R. KELLUM AND B. D. GARRETT
}

\begin{abstract}
A blocking set of a function $f$ is a closed set which does not intersect $f$ but which intersects each continuous function with domain the same as $f$. It is shown that for each function which is not almost continuous, there exists a minimal blocking set. Using this property it is shown that there exists an almost continuous function with domain $[0,1]$ which is a $G_{\delta}$ set but is not of Baire Class 1 , and that there exists an almost continuous function dense in the unit square.
\end{abstract}

Unless otherwise stated, all functions considered are real functions with domain a closed and bounded subset of the real line, $R$. No distinction is made between a function and its graph. If the function $f$ is a connected subset of the plane, $f$ is simply said to be a connected function. If each open set containing $f$ also contains a continuous function with the same domain as $f$, then the function $f$ is said to be almost continuous. Stallings [7] showed that if the function $f$ is almost continuous and has connected domain, then $f$ is connected. He stated as an open question the following. Is each connected function almost continuous? This question was answered in the negative by several authors ([1], [2], [4], [6]).

In [4], Jones and Thomas showed that there exists a function which is not of Baire Class 1 , is a $G_{\delta}$ set, is connected but is not almost continuous. In Example 1 of the present paper, it is shown that there exists such a function which is almost continuous. Example 2 shows that there exists an almost continuous function which is a dense subset of the unit square. In [1], Brown gave an example of a connected but not almost continuous function dense in $[0,1] \times R$.

Suppose $M$ is a subset of the plane. The $X$-projection of $M$ is denoted by $(M)_{X^{-}}$. The $Y$-projection of $M$ is denoted by $(M)_{Y}$. If $K \subset(M)_{X}, M_{K}$ denotes the part of $M$ with $X$-projection $K$.

The function $f$ is said to be of Baire Class $l$ if $f$ is the pointwise limit of a sequence of continuous functions.

Suppose that $D$ is a set containing the function $f$. The statement that the subset $C$ of $D$ is a blocking set of $f$ relative to $D$ means that $C$ is closed

Received by the editors June 15, 1971.

AMS 1970 subject classifications. Primary 54C10, 54C30, 26A15; Secondary 26A21.

Key words and phrases. Connected function, connectivity function, almost continuous.

c American Mathematical Society 1972 
relative to $D . C$ contains no point of $f$, and $C$ intersects $g$ whenever $g$ is a continuous function such that $(g)_{X}=(f)_{X}$ and $g \subset D$. If no proper subset of $C$ is a blocking set of $f$ relative to $D, C$ is said to be a minimal blocking set of $f$ relative to $D$.

THEOREM 1. Suppose the function $f$ has closed and bounded domain $H$, range $K$, and is not almost continuous. Then there exists a minimal blocking set $C$ of $f$ relatice to $H \times K$. Further, $(C)_{X}$ is contained in a component of $H$ and, if $K$ is connected, $\mathrm{Cl}\left((C)_{X}\right)$ is connected.

Proof. Since $f$ is not almost continuous, there exists an open set $D$ containing $f$ such that $D$ contains no continuous function with domain $H$. Then $(H \times K)-(D \cap(H \times K))$ is a blocking set of $f$ relative to $H \times K$. Now, suppose $U$ is a monotonic collection of blocking sets of $f$ relative to $H \times K$ and $g$ is a continuous function with $(g)_{X}=H$ and $g \subset H \times K$. Then $g$ intersects each member of $U$. So, the collection of sets $g \cap N$ where $N$ is in $U$ is a monotonic collection of closed and bounded subsets of the plane and must have a common part. Thus, the sets in $U$ have an intersection which is a blocking set of $f$ relative to $H \times K$. It follows that there exists a minimal blocking set $C$ of $f$ relative to $H \times K$.

Assume $(C)_{X}$ intersects more than one component of $H$. Let $s$ be an open segment contained in $R-H$ such that $(C)_{X}$ has a point to the left of $s$ and a point to the right of $s$. Denote by $N$ the part of $H$ to the left of $s$ and by $M$ the part of $H$ to the right of $s$. By the minimality of $C$, there exist continuous functions $g$ and $h$, each of which has domain $H$ and range a subset of $K$, such that $g$ contains no point of $C_{X}$ and $h$ contains no point of $C_{Y Y}$. Then $g_{X} \cup h_{Y Y}$ is a continuous function which has domain $H$. range a subset of $K$ and contains no point of $C$, a contradiction.

Assume that $K$ is connected but that $\mathrm{Cl}\left((C)_{X}\right)$ is not. Let $s=(m, n)$ be a segment in $R-C l\left((C)_{X}\right)$ such that $(C)_{X}$ has a point on either side of $s$. As in the previous paragraph, there exist continuous functions $g$ and $h$ such that $(g)_{X}=H \cap(-\infty, m],(h)_{X}=H \cap[n, \infty), g \subset H \times K, h \subset H \times K$, and neither of $g$ and $h$ intersects $C$. Denote by $L$ the line segment with endpoints $(m, g(m))$ and $(n, h(n))$. Since $K$ is connected $(L)_{Y} \subset K$. Thus $g \cup L \cup h$ is a continuous function with domain $H$, range $K$ and contains no point of $C$, a contradiction.

NoTE. For the set $C$ defined in Theorem $1,(C)_{X}$ is clearly nondegenerate. Thus, if $f$ is a real function with domain a nowhere-dense bounded closed set $H$, each component of $H$ is degenerate and $f$ is almost continuous.

We now give an example of an almost continuous function which is a $G_{o}$ set but which is not of Baire Class 1 .

EXAMPLE 1. Denote by $K$ the Cantor middle-thirds set in $I=[0,1]$ and by $J=\left(e_{1}, e_{2}, e_{3}, \cdots\right)$ the set of endpoints of complementary segments 
of $K$. Suppose $f$ is the function with domain $I$ and range $[-1,1]$ such that:

(1) If $z$ is in $I-K$ and $(m, n)$ is the component of $I-K$ containing $z$, then $f(z)=\sin (1 /(m-z)(n-z))$,

(2) if $z$ is in $K-J, f(z)=1$,

(3) if $z$ is in $J, f(z)=1 / r$, where $z$ is $e_{r}$.

Since $f_{K}$ is totally discontinuous. $f$ is not of Baire Class 1. Clearly $f_{I-K}$ and $f_{K-J}$ are $G_{\delta}$ sets. Since no point of $f_{J}$ is a limit point of $f_{J}, f_{J}$ is a $G_{\delta}$ set $\left[5\right.$, p. 68], so $f$ is a $G_{\delta}$ set.

Now, assume that $f$ is not almost continuous. By Theorem 1, there exists a minimal blocking set $C$ of $f$ relative to $I \times[-1,1]$ and $(C)_{X}$ is connected and nondegenerate. If $s$ is a complementary segment of $K$, $f_{s}$ is continuous. Clearly, $(C)_{X}$ contains a complementary segment $s=$ $(m, n)$ of $K$. By the minimality of $C$, there exist continuous functions $g$ and $h$, each of which has range a subset of $I$, neither of which intersects $C$, such that $(g)_{X}=[0, m]$ and $(h)_{X}=[n, 1]$.

Each of $(m, g(m))$ and $(n, h(n))$ is a limit point of $f_{s}$. Denote by each of $L_{1}$ and $L_{2}$ a line segmeni which contains ne point of $C$ such that $L_{1}$ has endpoints $(m, g(m))$ and $\left(z_{1}, f\left(z_{1}\right)\right)$ and $L_{2}$ has endpoints $(n, h(n))$ and $\left(z_{2}, f\left(z_{2}\right)\right)$ where $m<z_{1}<z_{2}<n$. Then

$$
g \cup L_{1} \cup f_{\left[z_{1}, z_{2}\right]} \cup L_{2} \cup h
$$

is a continuous function with domain $I$, range a subset of $[-1,1]$ and contains no point of $C$, a contradiction. Therefore $f$ is almost continuous.

The following example shows there exists an almost continuous function dense in $I^{2}$.

EXAMPLE 2. Denote by $U$ the set of all upper semicontinuous functions with domain $I$ and range a subset of $I$. Then $U$ is equally numerous with $R[3$, p. 140]. Denote by $V$ a collection of mutually exclusive dense subsets of $I$ whose union is $I$ such that $V$ is equally numerous with $R$. Let $T$ be a reversible transformation with domain $V$ and range $U^{\prime}$.

Suppose $f$ is the function with range and domain $I$ such that $f(z)=g(z)$ where $g=T(N)$ and $N$ is the member of $V$ containing $z$. Assume $f$ is not almost continuous. Then there exists a minimal blocking set $C$ of $f$ relative to $I^{2}$, and $(C)_{X}$ is connected and nondegenerate. Then the function $u$ such that $u(z)=\max \left(C_{z}\right)_{Y}$ is an upper semicontinuous function with domain $(C)_{X}$. Thus, $u$ and $f$ intersect. Then $f$ and $C$ intersect, a contradiction. Therefore, $f$ is almost continuous, and clearly, is dense in the unit square.

\section{BIBLIOGRAPHY}

1. Jack B. Brown, Connectivity, semi-continuity, and the Darboux property, Duke Math. J. 36 (1969), 559-562. MR 39 \#7568. 
2. J. L. Cornette, Connectivity functions and images on Peano continua, Fund. Math. 58 (1966), 183-192. MR 33 \#6600.

3. C. Goffman, Real functions, Rinehart, New York, 1953. MR 14, 855.

4. F. B. Jones and E. S. Thomas, Jr., Connected $G_{\delta}$-graphs, Duke Math. J. 33 (1966), 341-345. MR 33 \#702.

5. R. L. Moore, Foundutions of point set theory, rev. ed., Amer. Math. Soc. Colloq. Publ., vol. 13, Amer. Math. Soc., Providence, R.I., 1962. MR 27 \#709.

6. J. H. Roberts, Zero-dimensional sets blocking connectivity functions, Fund. Math. 57 (1965), 173-179. MR 33 \#3270.

7. J. R. Staliings, Fixed point theorems for connectivity maps, Fund. Math. 47 (1959), 249--263. MR 22 \#485.

Department of Mathematics, University of Alabama, University, Alabama 35486

Current address (K. R. Kellum): Department of Mathematics, Miles College, Birmingham, Alabama 35208 